\title{
DISEÑO DE UNIDADES DIDÁCTICAS EN EL ÁREA DE CIENCIAS EXPERIMENTALES
}

\author{
SÁNCHEZ BLANCO, G. y VALCÁRCEL PÉREZ, M.V. \\ Departamento de Didáctica de las Ciencias Experimentales. Escuela Universitaria de Magisterio. \\ Campus Espinardo. 30100 Murcia.
}

\section{SUMMARY}

This study puts forward some planning strategies for the teaching of Science based on a constructivist approach for the teaching/learning process. These strategies are shown in a model for the design of teaching units which includes five interdependent tasks: scientific analysis, pedagogical analysis, selection of objectives, selection of pedagogical strategies and selection of evaluation strategies. The first two aim at the reflection and estimation of contents and constrains of the teaching/learning processes. The other three are concerned with teachers' decision making and the explicitness of their teaching plans, as well as with their classroom performance in order to achieve them.

\section{INTRODUCCIÓN}

La preparación de una lección es una tarea que ha de acometer el docente. ¿Qué contenidos incluyo en la lección? ¿Por dónde comienzo su desarrollo? ¿Qué experiencias de laboratorio debo hacer?... son algunas de las preguntas que frecuentemente se hace un docente. Es una preocupación compartida por los profesores que su trabajo requiere una planificación, aunque diferentes estudios realizados (Clark y Peterson 1990) sobre cómo Ilevan a cabo los profesores este proceso han puesto de manifiesto que, en general, es considerado como una lista flexible de acciones centradas fundamentalmente en los contenidos y las actividades de enseñanza, estando implícitos los objetivos o metas a lograr. Se resaltan diferencias en las fuentes que utilizan los profesores para tomar decisiones, destacando el diagnóstico de los alumnos y los materiales curriculares, los cuales llegan a constituir el inicio y final de la planificación.

En nuestro contex to educativo, Salinas (1990) diferencia tres niveles de planificación -burocrática, organizativa y progresiva- tanto por la estrategia que supone como por la utilidad que tiene para el profesor.

Aunque también es manifiesto que las dificultades y estrategias son diferentes para el profesor novel o experto, la utilización de un libro de texto o la de varios simultáneamente para la elaboración de apuntes, y la preparación de actividades prácticas (ejercicios, proble. mas, experiencias... ) es algo a lo que todos nos hemos visto abocados, con mayor o menor convencimiento de que fuera io más adecuado, y que sigue siendo habitual para muchos docentes.

Obligada referencia requiere la reforma del sistema educativo. La reciente aprobación de la LOGSE plantea un difícil reto al profesorado de este país, pues, to quiera o no, de él depende su implantación real. Creemos que no es tarea fácil porque cada profesor desde su área de conocimiento tendrá que aportar su saber para concretar el Diseño Curricular Base (MEC 1989) en proyectos de centro que recojan los currículos disciplinares o interdisciplinares que consideren pertinentes. El paso más difícil y delicado, por las repercusiones que tendrá en el logro de los objetivos generales de cada área, será cuando los profesores tengan que planificar y secuenciar las Unidades Didácticas (UD) que configuran el currículom. En este sentido, creemos que el modelo que se presenta puede ser útil al profesor, pues le indica un procedimiento para la planificación de la enseñanza que tiene en consideración las referencias que fundamentan el DCB. 


\section{MODELO PARA LA PLANIFICACIÓN DE LA ENSENANZA}

La planificación de una lección está condicionada por una serie de factores (tipo de contenidos, número de alumnos por aula, experiencias previas del profesor y los alumnos...) sobre los que no es fácil ponerse de acuerdo si intentáramos jerarquizarlos por lo decisivo de su incidencia. Sin embargo, sí que creemos posible establecer una relación de ellos con tres referencias que consciente o inconscientemente tiene cualquier profesor de ciencias: su formación científica, su formación didáctica y su modelo educativo.

La Didáctica de las Ciencias Experimentales se nutre por las ideas ejes que definen la Naturaleza de las Ciencias y la Naturaleza del proceso de Enseñanza / Aprendizaje (E/A) (Linn 1987, Hewson y Hewson 1988, Aliberas et aI. 1989). Son, por tanto, estas ideas las referencias obligadas que ha de tener el profesor para planificar su enseñanza y, por consiguiente, éstas han sido las que han inspirado el modelo que presentamos. Obviamente, no han sido éstas nuestras únicas referencias, pues como profesores difícilmente podemos sustraernos de las teorías personales y creencias educativas configuradas durante unos cuantos años de práctica docente. En el mapa «Didáctica de Ciencias» (Fig. 1) mostramos la relación entre el tipo de competencias que requiere un profesor de Ciencias y las acciones que ha de acometer para planifjcar su enseñanza.

Las acciones que se recogen (análisis científico, análisis didáctico, objetivos, estrategias didácticas y evaluación) son las cinco tareas incluidas en el modelo que proponemos y describimos de manera resumida en el cuadro «Modelo para el diseño de UD» (Fig. 2). Perseguimos un doble propósito: proporcionar las referencias teóricas que puedan fundamentar la toma de decisiones del profesor en la planificación y facilitar un procedimiento para abordar cada una de estas tareas.

Existen diversas vías para diseñar un currículo en sus diferentes niveles de concreción (Coll 1987), al considerar que en todo proceso de E/A están presentes tres elementos básicos, el contenido, los resultados esperados y las actividades, pudiendo contemplarse cada uno

Figura 1

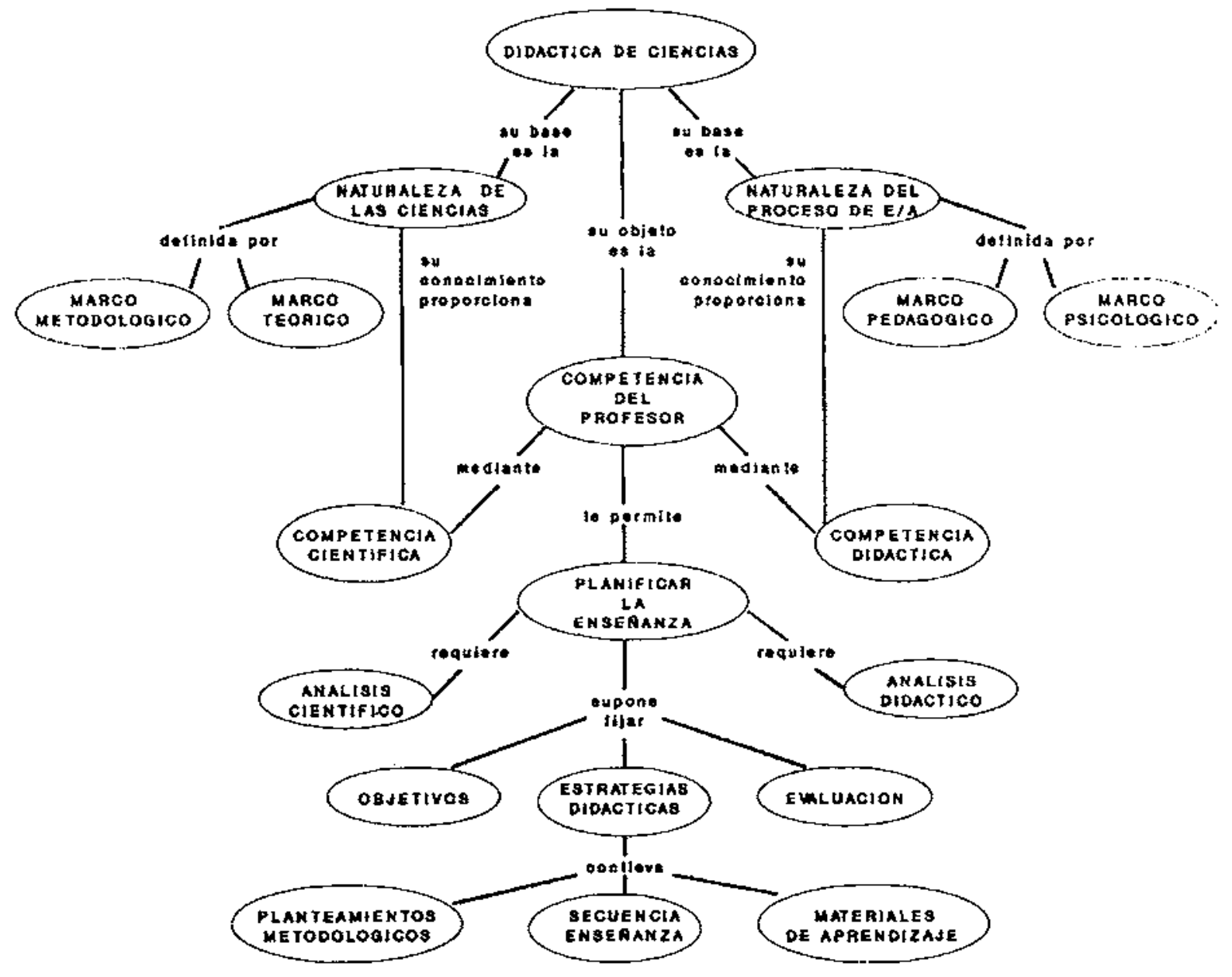


Figura 2

Modelo para el diseño de unidades dídácticas.

\section{OBJETIVOS}

\section{PROCEDIMIENTOS}

\section{ANÁLISIS CIENTÍFICO}

a) La reflexión y actualización científica del profesor

b) La estructuración de los contenidos
1) Seleccionat los contenidos
2) Definir el esquema conceptual
3) Delimitar procedimientos científicos
4) Delimitar actitudes científicas

\section{ANÁLISIS DDDÁCTICO}

a) La delimitación de los condicionamientos del proceso de E/ A: adecuación al alumno
1) Averiguar las ideas previas de los alumnos

2) Considerar las exigencias cognitivas de los contenidos

3) Delimitar implicaciones para la enseñanza

\section{SELECCIÔN DE OBJETIVOS}

a) La reflexión sobre los potenciales aprendizajes de los alumnos

b) El establecimiento de referencias parael proceso de evaluación
1) Considerar conjuntamente el $\mathrm{AC}$ y el $\mathrm{AD}$

2) Delimitar prioridades y jerarquizarlas

\section{SELECCIÓN DE ESTRATEGLAS DIDÁCTICAS}

a) La determinación de las estrategias a seguir para el desarrollo del tema

b) La definición de tareas a realizar por profesor y alumnos

1) Considerar los planteamientos metodológicos para la enseñza
2) Diseñar la secuencia global de enseñanza
3) Seleccionar actividades de enseñanza

4) Elaborar materiales de aprendizaje

\section{SELECCION DE ESTRATEGIAS DE EVALUACIÓN}

a) La valoración de la unidad diseñada

b) La valoración đel proceso de enseñanza y de los aprendizajes de los alumnos

\begin{abstract}
1) Delimitar el contenido de la evaluación
2) Determinar actividades y momentos del desarrolio del tema
\end{abstract}

3) Diseñar instrumentos para la recogida de información de ellos desde el punto de vista de la enseñanza (profesor) o del aprendizaje (alumno). El último nivel de concreción del currículo es el diseño de UD, y aún siendo legítimas cualquiera de las vías anteriores, optamos por una vía mixta que contempla el análisis de los posibles contenidos de enseñanza y el análisis de los potenciales aprendizajes de los alumnos. Por ello el modelo de planificación que proponemos comienza con dos tareas previas a la delimitación de los objetivos de la UD: Análisis Científico y Análisis Didáctico. La necesidad de los mismos como paso previo en la planificación de la enseñanza está cada vez más justificada (Valcárcel et al. 1990), pero la importancia de ambos e interdependencia dificultan su jerarquización. Siendo conscientes que el proceso de planificación de un profesor no es en absoluto lineal (Clark y Yinger 1979), pues es difícil sustraerse a referencias y condicionantes implicados en las diferentes tareas, proponemos como proceso analítico en primer lugar el análisis científico, por diversas consideraciones centradas en tres puntos: a) La formación inicial del profesor de Ciencias de la enseñanza secundaria obligatoria está claramente orientada a la adquisición de conocimientos científicos y, por tanto, es su referencia más inmediata.

b) El profesor es el mediador del conocimiento entre la Ciencia y el alumno. Los conceptos, procedimientos y actitudes implicados en el desarrollo de la UD serán las referencias comunes a los sujetos implicados en el proceso de comunicación inherente a la acción educativa. Dado que las estructuras sustanciales de las disciplinas son un elemento fundamental para la educación (Coll y Solé 1987), la decisión sobre qué conocimiento compartir es algo prioritario.

c) Los problemas relativos al aprendizaje escolar están condicionados en gran medida por la especificidad del contenido de enseñanza (Coll 1988). Pero, además, si compartimos aquellas teorías que sustentan que el aprendizaje conleva un cambio conceptual (Posner et 
al. 1982, Osborne y Wittrock 1985, Pozo 1990), parece lógico que conozcamos y reflexionemos sobre el significado y las relaciones de los conceptos implicados, pues hacia ahí hemos de dirigir los aprendizajes de los alumnos $y$, por lo tanto, deben inspirar nuestras decisiones sobre la selección de objetivos, estrategias didácticas o de evaluación. De igual modo deben dirigir las indagaciones que hagamos sobre las experiencias e ideas de los alumnos, así como las implicaciones didácticas que de ellas se deriven.

\section{TAREAS PARA LA PLANIFICACIÓN}

Vamos a referirnos de una manera más precisa a las tareas incluidas en el modelo que presentamos incidiendo tanto en sus objetivos como en los procedimientos que se proponen para abordarlas.

I. El objetivo del análisis científico es doble: la estructuración de los contenidos de enseñanza y la actualiza- ción científica del profesor, derivada del proceso de consulta y reflexión sobre el propio conocimiento científico incluido en la UD.

La selección del contenido de enseñanza ha de ser coherente con las actuales concepciones sobre la Naturaleza de la Ciencia (Hodson 1988a, 1988b, Gil 1986) y, dado que la naturaieza de un conocimiento está determinado por su epistemología, debemos atender a lo que caracteriza la investigación científica.

Actualmente, la investigación se concibe como un proceso cíclico que comienza y termina con el planteamiento de problemas que originan los conocimientos existentes. Se realza el papel que el conocimiento inicial juega en la producción del conocimiento. Se relativiza el papel que juega en una investigación la observación y experimentación en favor de otros procesos como la emisión de hipótesis o el diseño experimental. Se admite que el trabajo de los científicos tiene unas peculiaridades que se describen mediante un conjunto de procesos, que se pueden clasificar en básicos e integrados. También se

Figura 3

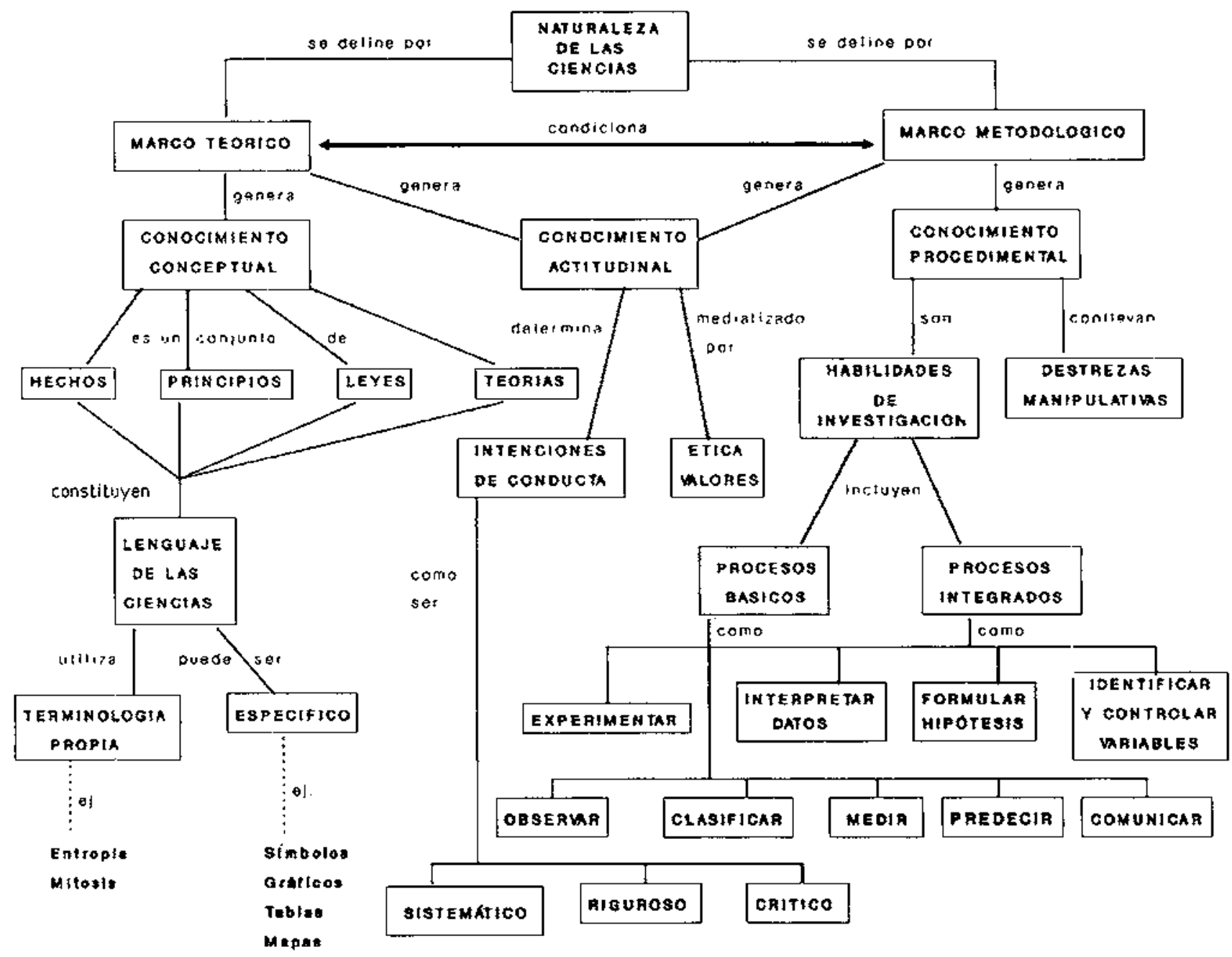


admite que el conocimiento científico es un conocimiento estructurado y cambiante, construido por colectivos o comunidades de científicos que trabajan dentro de un mismo paradigma o programa de investigación (Chalmers 1982).

La diferenciación del conocimiento o del contenido de enseñanza, en conceptual, actitudinal y procedimental es con carácter analítico y fundamentalmente por motivos pedagógicos (Coll et al. 1992). Lógicamente, estos conceptos siempre han estado presentes en la Ciencia aunque con otros términos. Así, tradicionaimente diferenciábamos contenidos científicos o conocimientos, habilidades de investigación -incluyendo las manipulativas- y actitudes. En el mapa «Naturaleza de las Ciencias" (Fig. 3) se plantea este triple aspecto y los contenidos que hay que trabajar en relación con ellos. El hecho de diferenciar este triple aspecto del conocimiento científico nos permite tomar conciencia de las diferentes facetas de la enseñanza de las ciencias, no debiendo por tanto ocuparse sólo de una de ellas, generalmente la conceptual. Pero existe el peligro de una interpretación errónea que lleve al profesor a creer que cada tipo de contenido es suceptible de trabajarse independientemente. No podemos olvidar que el conocimiento científico es único y las estrategias de aprendizaje que adoptemos deben integrar los tres contenidos.

Es importante comprender las relaciones existentes entre los elementos que hemos destacado del conocimiento conceptual (hechos, principios, leyes y teorías) y entre los del procedimental (experimentar, interpretar datos...), así como la de todos entre sí, pues dado que la enseñanza se debe hacer de manera gradual es necesario que explicitemos el sentico que tienen esos contenidos para adquirir un conocimiento. Así, la realización de una gráfica, la medida đe una magnituđ, el estudio de una ley, el aprendizaje de unos símbolos o fórmulas, por citar algunas cosas usuales en la enseñanza de las ciencias, debe tener para el alumno un sentido que vaya más allá que el de superar las dificultades que en sí mismo tiene cualquier aprendizaje.

La importancia del marco teórico en la construcción de la ciencia y el hecho de que sea un conocimiento estructurado sujeto a ampliaciones y modificaciones lo destacamos no sólo por las implicaciones que deberemos considerar para la enseñanza de las Ciencias, sino por ser el que fundamenta el procedimiento que proponemos para el análisis científico.

La estructuración de los contenidos de la UD es uno de Ios objetivos del análisis científico y debe referirse a un marco teórico o esquema conceptual concreto. La UD la definimos por los contenidos que el profesor considere necesarios para proporcionar al alumno un esquema conceptual científico sobre el objeto de estudio. Como profesores no nos interesa que el alumno adquiera uno o muchos conceptos aislados, sino que los utílice en un entramado conceptual más complejo que le permita explicar hechos o fenómenos de una manera similar a como lo hace la Ciencia. Puesto que el desarrollo de un esquema conceptual sólo es posible mediante el establecimiento de relaciones entre conceptos y la ampliación de sus significados, la delimitación del esquema es importante.

La amplitud y duración de una UD dependerá de la amplitud y complejidad del esquema conceptual que decidamos desarrollar.

En el procedimiento que proponemos, se comienza seleccionando el contenido cientiffico en términos generales, lo que vendría a constituir un índice de los hechos de interés. Incluir contenidos que consideren aspectos relativos a la identificación, interpretación y aplicación del objeto de estudio es un criterio de selección coherente con la Naturaleza de la Ciencia. Los sistemas materiales (el agua, Ios alimentos, los circuitos eléctricos...), fenómenos (la flotación, los cambios de estado, la fotosíntesis...) o ambos aspectos conjuntamente (las soluciones y el proceso de disolución, el aparato digestivo y la digestión, circuitos eléctricos y electricidad...) son objetos de estudio en la enseñanza de la Ciencia.

Decidir qué contenidos pueden ser necesarios para la identificación supondrá responder a las preguntas: ¿qué es? o ¿qué ocurre? Este contenido tiene un carácter descriptivo y nos debe permitir conocer aquellas propiedades o funciones características. Por tanto, en relación con el objeto de estudio debemos seleccionar aquellos hechos, conceptos, principios o leyes que consideremos relevantes para ese fin.

Para los contenidos de interpretación debemos responder a las preguntas: ¿por qué es así? o ¿por qué ocurre de este modo? Es un contenido que tiene un carácter explicativo y nos debe permitir ir más allá đe los aspectos perceptivos. Debemos seleccionar aquellas teorías o modelos que expliquen el fenómeno o el comportamiento del sistema.

Seleccionar los contenidos referidos a la aplicación, como tranferencia a otras situaciones, supondrá responder a las preguntas ¿para qué sirve ese conocimiento? ¿ ¿qué nos puede explicar? Es un contenido que tiene un carácter funcional y predictivo, y nos debe permitir mostrar la relación entre la ciencia, la tecnología y la sociedad. Problemas cotidianos y domésticos de diversa índole, problemas relativos al medio ambiente, a la salud, procesos industriales, actividades lúdicas... en relación con el objeto de estudio deben incluirse en la unidad.

Una vez seleccionados los contenidos de la UD, la explicitación del esquema conceptual de la misma nos permite delimitar los conceptos y relaciones más relevantes de dichos contenidos. Un esquema conceptual puede explicitarse mediante «mapas de conceptos» (Novak y Gowin 1988). Dependiendo de la complejidad y amplitud del esquema, necesitaremos uno o más mapas. Es importante recordar que éstos son tan sólo representaciones de los esquemas conceptuales, pues las limitaciones de la técnica tan sólo permiten resaltar los conceptos y relaciones más relevantes. Creemos que el uso de esquemas conceptuales es habitual entre el profesorado, aunque la técnica que utilice para representarlos (guiones, resúmenes...) sea más rudimentaria que los mapas. Aunque existen otras técnicas para representar esque- 
mas conceptuales (redes de conceptos, diagramas de flujo, diagramas de llaves...), nosotros pensamos que los mapas tienen un especial interés por ser un instrumento educativo basado en la teoría del aprendizaje significativo de Ausubel.

La delimitación de los contenidos procedimentales debe supeditarse al entramado conceptual al que queramos llegar, pues sólo así estos nuevos contenidos tendrán significado científico, perdiendo su carácter anecdótico (experiencias inconexas, descontextualizadas...). Para identificar los procedimientos que pueden estar implicados, consideramos útil el marco de reflexión de las «cuatro preguntas» que propone Gowin (Novak y Gowin 1988) para el análisis de las tareas de enseñanza. Su adaptación para seleccionar los contenidos procedimentales de la UD nos lleva a proponer, en relación con un hecho del objeto de estudio, la siguiente secuencia:

1. ¿Cuál es el conocimiento al que pretendemos llegar con los procedimientos que seleccionemos? («afirmaciones de conocimiento").

2. ¿A qué preguntas o problemas da respuesta ese conocimiento? («preguntas determinantes»).

3. ¿Qué conceptos están implícitos en esas preguntas y debe conocer el alumno para encontrarle sentido al estudio del hecho seleccionado? («conceptos claves pertinentes»).

4. ¿Cuáles son los procedimientos que se requieren para responder a las preguntas determinantes y llegar a las afirmaciones de conocimiento que deseamos que aprendan nuestros alumnos? («métodos de investigación»).

Dado que los aspectos a considerar en un esquema conceptual pueden ser diversos y, por tanto, diversas también las afirmaciones de conocimiento a las que Ilegar, deberemos seleccionar aquéllas que nos ofrezcan mayores posibilidades en la delimitación de los procedimientos. Se trata, en consecuencia, de delimitar de los procesos básicos e integrados con los que se aborda la resolución đe problemas científicos, aquéllos que sean más relevantes para los aprendizajes de los conteniđos conceptuales de la UD.

Es importante señalar que, puesto que los contenidos procedimentales han de permitir al alumno adquirir conceptos, desarrollar actitudes y llegar en último término a alcanzar un alto grado de autonomía en el aprendizaje (Hernández 1989, Sanmartí et al. 1990), son a la vez contenidos de enseñanza y mediadores del proceso de aprendizaje.

La delimitación de los contenidos actitudinales se facilita una vez realizados los conceptuales y procedimentales, pues con éstos se van a generar unas actitudes determinadas $o$, más concretamente, valores y normas. Las actitudes a su vez guían los procesos perceptivos y cognitivos que conducen al aprendizaje de contenidos conceptuales y procedimentaies (Sarabia 1992).
Los contenidos de aplicación seleccionados nos proporcionan especialmente una referencia para las actitudes, ya que la relación Ciencia, Tecnología, Sociedad es una fuente importante de actitudes hacia la Ciencia y el trabajo científico. Del mismo modo, los procedimientos seleccionados tienen también especial relevancia en la construcción de actitudes científicas y hacia la Ciencia, así como en la utilización de normas. Otros contenidos actitudinales estarán más relacionados con formas de trabajo en el aula.

II. Una vez estructurados los contenidos de la UD desde una perspectiva científica, aunque no exenta de la intencionalidad didáctica con la que el profesor realiza la tarea anterior, proponemos el análisis didáctico con el objetivo de delimitar los condicionantes del proceso de E/A.

Dentro de esta tarea vamos a centrarnos en la capacidad cognitiva del alumno por ser este factor determinante de lo que es capaz de hacer y aprender en cualquier situación. Obviamente, no es el único condicionante del proceso de E/A, pues inciden otros factores como la competencia profesional del profesor, los hábitos de trabajo de los alumnos, sus actitudes e intereses, el ambiente del aula, los recursos del centro... generadores de problemas a los que no es posible dar respuesta puntualmente. Algunos de ellos se considerarán globalmente cuando nos planteemos las estrategias de enseñanza y se realice la selección de actividades.

Dos referencias podemos considerar como indicadores de la capacidad cognitiva del alumno: sus conocimientos previos sobre el tema y el nivel del desarrollo operatorio donde se encuentran los alumnos (Coll 1986) en relación con las habilidades intelectivas necesarias para la comprensión de la Ciencia. EI diferenciar ambos factores responde a una exigencia analítica y epistemológica, pues proceden de teorías distintas (teoría del aprendizaje y teoría del conocimiento), aunque para nosotros tienen en común el ser útiles por explicar las dificultades que los alumnos pueden tener para el aprendizaje de los contenidos de enseñanza seleccionados.

Lo que el alumno sabe sobre un contenido determinado, o en relación con una tarea de aprendizaje constituyen sus conocimientos previos y es manifiesta su importancia en la realización de aprendizajes significativos (Posner et al. 1982, Nusbaum y Novik 1982, Osborne y Wittrock 1985, Driver 1986). Lo que proponemos para analizar y considerar este referente es partir del esquema conceptual que define la UD, y que deseamos compartir con el alumno, e indagar en los conocimientos previos de los alumnos sobre los conceptos y relaciones más relevantes del mismo, así como sobre aquellos conceptos que sin ser contenidos de la UD constituyen requisitos previos del aprendizaje de los nuevos conocimientos.

La importancia de conocer las ideas de los alumnos no radica sólo en detectar errores sino, y con el mismo interés, también sus aciertos, pues ambos tienen la misma relevancia en la estructura cognitiva del alumno y ambos serán las herramientas conceptuales que utilice para hacer inteligible la nueva información que incorpore- 
mos en el proceso de enseñanza. Así, al planificar la enseñanza de una UD, debemos conocer lo que nuestros futuros alumnos saben, lo cual plantea en cierto modo un problema, pues es posible que ni los conozcamos. Las aportaciones de la investigación educativa palian este problema en gran parte, al conocer sus características generales y disponer de un amplio inventario de ideas sobre contenidos usuales en la enseñanza de las ciencias, tanto de los conocimientos previos a la enseñanza como de su evolución con el proceso de E/A (Hierrezuelo y Montero 1989, Driver et al. 1989, Pozo et al. 1991).

Bien para contrastar la información bibliográfica en nuestro contex to educativo o bien porque sea necesario indagar en temas de interés insuficientemente tratados, puede ser necesario realizar exploraciones en nuestras aulas. Diversas son las técnicas y estrategias utilizadas (entrevistas, cuestionarios, pruebas experienciales, tareas razonadas...) para detectar las ideas de los alumnos (García-Estañ et al.1988, Giordan y Vecchi 1988, Driver et al. 1989, Pozo 1992). En cualquier caso, las indagaciones, bibliográficas o en el aula, deben estar dirigidas hacia aquellos contenidos seleccionados como relevantes desde la perspectiva científica y sobre los que presuponemos que los alumnos tendrán serias dificultades bien por nuestra experiencia docente o por la información recabada.

Nos hemos referido al estadio de desarrollo operatorio como el otro factor determinante de la capacidad cognitiva del alumno. Nos parecen relevantes las aportaciones de Shayer y Adey (1984), pues nos proporcionan un marco explicativo (Taxonomías I y II) de utilidad para el objeto de esta tarea. Concretamente, nos describen cómo se desarrollan diferentes esquemas de conocimiento necesarios para la comprensión de la ciencia (conservación, equilibrio de sistemas, control de variables...) y cómo interacciona el niño con el mundo físico que le rodea (actitud investigadora, razones de los hechos, uso de modelos...). Desde este marco podemos contrastar las exigencias cognitivas del contenido de enseñanza seleccionado con las habilidades intelectivas y posibilidades de razonamiento de los alumnos para el procesamiento de la información, lo que nos permitirá disponer de explicaciones más consistentes de muchas dificultades de los alumnos a las que somos sensibles los profesores como consecuencia de nuestra experiencia docente (cálculo numérico, identificación de variables, capacidad de abstracción...).

Puesto que los diferentes tópicos científicos que constituyen los programas básicos de Ciencias (Secundaria Obligatoria y Bachillerato) pueden presentarse en sucesivos niveles de complejidad y desarrollo conceptual, Ia información procedente de las exigencias cognitivas de los contenidos nos permitirá valorar la conveniencia de abordartos con un nivel de diferenciación conceptual determinado o la manera de abordarlos de forma que sea posible su comprensión. Para nosotros esta valoración debe hacerse más que con un sentido restrictivo con uno progresivo que favorezca el aprendizaje y contribuya al desarrollo de las habilidades intelectivas deseables en el estudiante de ciencias.
Lógicamente, no sólo se trata de identificar los condicionantes que el alumno tiene para aprender, sino que es necesario establecer conclusiones pertinentes para el proceso de planificación y, por tanto, dirigidas a la toma de decisiones sobre la enseñanza. En los siguientes aspectos deben centrarse nuestras conclusiones: $a$ ) el punto de partida del esquema conceptual que queremos desarollar; b) los conceptos más problemáticos; $c$ ) la adecuación de los procedimientos implicados; $d$ ) Ios objetivos prioritarios relativos al aprendizaje del alumno;e) las actividades que hemos de seleccionar para favorecer el proceso de aprendizaje; $f$ ) las referencias que debemos considerar para la evaluación de los aprendizajes đe los alumnos.

Para terminar, queremos puntualizar que el considerar tanto Ias ideas de los alumnos como sus habilidades de razonamiento no nos dan una solución única al problema del diseño de la UD o, dicho de otro modo, no nos garantizan el éxito de nuestra enseñanza, pero sí constituyen un punto de partida importante para acercarnos a la solución de muchos problemas del aprendizaje que se plantean en el aula y una posible explicación a las dificultades que el aprendizaje de la Ciencia plantea a los alumnos.

III. Realizados los análisis científico y didáctico, la siguiente tarea que proponemos es ia selección de objetivos. Es necesario que el profesor reflexione sobre los aprendizajes que desea favorecer en los alumnos, considerando simultáneamente los resultados de los análisis científico y didáctico, y que concrete en un conjunto de objetivos a conseguir sus intenciones educativas.

La reflexión y toma de decisiones del profesor deben estar guiadas por criterios coherentes con el nivel educativo y con el concepto de UD que proponemos. La selección de objetivos debe tener como referencia el nivel eđucativo en el que estamos y más concretamente la que nos proporcionan los Objetivos Generales de Área, que se definen en el $\mathrm{DCB}, 0$ aquéllos que se especifiquen para el Área de Ciencias en Ios Proyectos de Centro (Coll 1987, MEC 1989). Así mismo, debe estar dirigida por los contenidos relevantes (conceptuales, procedimentales y actitudinales) implicados en el desarrollo del «esquema conceptual» que define a la UD, más que por el logro de múltiples conceptos, leyes aisladas, habilidades puntuales....

El aborđar la selección de objetivos tras los análisis cientf́fico y didáctico debe servir para que contemplemos no sólo los contenidos científicos que queremos trabajar en el aula, sino también las experiencias previas y posibles dificultades de aprendizaje de los alumnos. Creemos que la confusión a menudo habitual entre contenidos y objetivos desde esta visión puede paliarse, pues es fácil comprender que un mismo contenido de enseñanza pueda plantearse con diferentes niveles de complejidad e implicando a los alumnos en diferentes estrategias de aprendizaje. El análisis didáctico nos ayuda a seleccionar objetivos más acordes con ambos puntos de partida: contenido-alumno y diferenciar por tanto contenido científico de objetivo didáctico. 
Uno de los problemas que surgen en esta tarea es el decidir el nivel de concreción con que formulamos los objetivos didácticos. Creemos que el nivel óptimo vendrá dado por la utilidad que tenga tanto para compartir con otros profesores las intenciones que dirigen la selección de estrategias didácticas y de evaluación de la unidad, como para dirigir el proceso de revisión de la UD tras su puesta en práctica en el aula. Sin embargo, buscar el equilibrio no es fácil, pues una concreción excesiva puede llevarnos a una lista demasiado amplia de objetivos y esta atomización puede hacernos perder la visión global que se requiere de la UD. En cualquier caso, debemos recordar que la formulación no ha de convertirse en una descripción de los resultados y conductas observables esperadas en nuestros alumnos, ya que no podemos olvidar que el carácter constructivo del aprendizaje supone la obtención de resultados particulares para cada alumno según sus peculiaridades $y$, por tanto, el desarrollo de capacidades en los alumnos no puede ser medido por determinadas conductas observables idénticas para todos.

IV. Para desarrollar una lección, el profesor debe adoptar unas normas de actuación ante las que espera que los alumnos respondan de una determinada manera. La selección de estrategias didácticas tiene por objeto el que estas normas de actuación sean eficaces para ellogro de los objetivos propuestos. Creemos necesario diferenciar, dentro de la estrategia didáctica de un profesor, sus planteamientos metodológicos, la secuencia de enseñanza, las actividades de enseñanza y los materiales de aprendizaje córno cuatro conceptos que nos permitirán comprender cómo se concreta la acción en el aula y nos serán útiles para la realización de esta tarea.

Los planteamientos metodológicos nos informan sobre las funciones que profesor y alumnos desempeñan en $\mathrm{el}$ proceso de E/A y están determinados por las teorías y creencias personales que el profesor sustenta, fundamentalmente, sobre la naturaleza de la Ciencia, la naturaleza del proceso de E/A y la función del sistema educativo. Aunque existen otros hechos determinantes de gran importancia como las limitaciones y posibilidades del profesor (Clark y Peterson 1990) destacamos los anteriores por considerarlos esenciales para reflexionar el origen de la metodología del profesor y estar relacionados con las tres primeras tareas que planteamos en el modelo de planificación.

Cada profesor, como individuo formado en un área científica, tiene sus propias creencias sobre lo que es la Ciencia y el papel que debe jugar en un sistema educativo, lo que se traduce en preferencias sobre el contenido (conocer leyes, manejo de aparatos, resolución de problemas...) a la hora de enseñar o la utilización de determinados recursos (explicación del profesor, laboratorio, vídeo...). Es necesario saber también que los planteamientos metodológicos bajo los que hemos sido instruidos, o cualquier otro, son consecuencia de una teoría de enseñanza y ésta a su vez es precedida por una teoría de aprendizaje. Querámoslo o no, por lo tanto, el profesor está transmitiendo una imagen, de la Ciencia y del proceso de E/A, que será aprehendida por sus alumnos y que éstos utilizarán para solucionar sus problemas escolares (preparación de exá- menes, realización de trabajos...), adquiriendo por tanto unas estrategias de aprendizaje directamente relacionadas con las teorías que, sobre la Ciencia y el proceso de $\mathrm{E} / \mathrm{A}$, «transmite» el profesor.

Scott (1989) describe las principales diferencias entre dos concepciones del proceso de E/A, la de transferencia de conocimientos y la de construcción de conocimientos. Una síntesis de estas diferencias es aportada por el equipo del proyecto CLIS (AAVV 1987b). El cuadro «Concepciones sobre el proceso de E/A» (Fig. 4) amplía esta sintesis destacando no sólo diferencias sino semejanzas. Pensamos que este análisis puede servir para que cada profesor reflexione sobre su situación personal y pueda plantearse estrategias que le permitan realizar aproximaciones hacia planteamientos más coherentes con la concepción constructivista.

Figura 4

Concepciones sobre el proceso de enseñanza-aprendizaje.

\begin{tabular}{|c|c|c|}
\hline Concepcion & TRANSFERENCIA & CONSTRUCCION \\
\hline $\begin{array}{l}\text { Papel del } \\
\text { profesor }\end{array}$ & Transmitir conocimientos & $\begin{array}{l}\text { Facilitar situaciones que } \\
\text { ayuden al alumno a } \\
\text { construir significados }\end{array}$ \\
\hline $\begin{array}{l}\text { Papel del } \\
\text { slumno }\end{array}$ & $\begin{array}{l}\text { Asimilar pasivamence la } \\
\text { informacion }\end{array}$ & $\begin{array}{l}\text { Construir activamente } \\
\text { significados }\end{array}$ \\
\hline $\begin{array}{l}\text { Mente del } \\
\text { alumno }\end{array}$ & $\begin{array}{l}\text { Vacias o con ideas } \\
\text { fácilmente reemplazables }\end{array}$ & $\begin{array}{l}\text { Ideas fuertemente } \\
\text { acomodadas basadas en } \\
\text { su experiencia }\end{array}$ \\
\hline $\begin{array}{l}\text { Dependencls } \\
\text { del } \\
\text { aprendizaje }\end{array}$ & $\begin{array}{l}\text { Situasiones externas } \\
\text { (profesor, clase, libros, } \\
\text { experimentos...) }\end{array}$ & $\begin{array}{l}\text { Situaciones externas y } \\
\text { experiencias e ideas } \\
\text { previas de los alumnos }\end{array}$ \\
\hline Aprendizzie & $\begin{array}{l}\text { Rellenar un recipiente } \\
\text { vacío }\end{array}$ & $\begin{array}{l}\text { Modificar, sustituir o } \\
\text { ampliar ideas/conceptos } \\
\text { existentes }\end{array}$ \\
\hline Copocimtento & $\begin{array}{l}\text { Algo que existe "fueta", } \\
\text { independiente de quien lo } \\
\text { conoce }\end{array}$ & $\begin{array}{l}\text { Algo que debe ser } \\
\text { construido por cada } \\
\text { individuo }\end{array}$ \\
\hline Evalugción & $\begin{array}{l}\text { El profesor controla el } \\
\text { proceso }\end{array}$ & $\begin{array}{l}\text { Profesor y alumno } \\
\text { controlan el proceso }\end{array}$ \\
\hline $\begin{array}{l}\text { Recursos y } \\
\text { actividades }\end{array}$ & \multicolumn{2}{|c|}{$\begin{array}{l}\text { Exposiciones del profesor, lecturas de textos, } \\
\text { audiovisuales, experiencias de laboratorio, resolucion } \\
\text { de problemas, cuestiones, trabajo individual y en } \\
\text { grupo... }\end{array}$} \\
\hline
\end{tabular}

Unos mismos planteamientos metodológicos pueden traducirse en acciones docentes diferentes (Gil y Martínez 1987, Driver 1988, Coscrove y Osborne 1991). Definir la secuencia de enseñanza es necesario para concretar cómo vamos a llevar al aula nuestros planteamientos metodológicos. Para ello debemos señalar las fases o etapas, incluidas en su desarrollo resaltando el objetivo u objetivos que persiguen. Con el cuadro «Secuencia de enseñanza» (Fig. 5), queremos reflejar la diferente intencionalidad de una misma secuencia, igual número y denominación de las fases en función de los planteamientos metodológicos. Independientemente de la terminología y el número de fases que utilicemos, lo importante al diseñar la secuencia de enseñanza es que distribuyamos el contenido seleccionado precisando cómo desarrollaremos el esquema conceptual de la UD, es decir đecidir cuál será el camino que seguiremos. Haber 
Figura 5

Secuencia de enseñanza.

\begin{tabular}{|c|c|c|}
\hline Concepción & TRANSFERENCIA & CONSTRUCCION \\
\hline $\begin{array}{l}\text { 1'Fase } \\
\text { Iniciacion }\end{array}$ & $\begin{array}{l}\text { a) Contextualizar la } \\
\text { nueva unidad en relación } \\
\text { con otros conocimientos } \\
\text { b) Organizar el contenido } \\
\text { que se va a desamollar } \\
\text { c) Motivar (interesar) al } \\
\text { alumno por el contenido }\end{array}$ & $\begin{array}{l}\text { a) Igual } \\
\text { b) Igual } \\
\text { c) Igual } \\
\text { d) Explicar (poner de } \\
\text { manifiesto semejanzas y } \\
\text { diferencias) las ideas } \\
\text { previas de los alumnos }\end{array}$ \\
\hline $\begin{array}{l}2 \text { Fase } \\
\text { Informacion }\end{array}$ & $\begin{array}{l}\text { a) Introducir nuevos } \\
\text { conocimientos } \\
\text { (conceptuales, } \\
\text { procedimentales, } \\
\text { actitudinales) }\end{array}$ & $\begin{array}{l}\text { a) Clarificar e } \\
\text { intercambiar ideas } \\
\text { previas } \\
\text { b) Exponer a los alumnos } \\
\text { a siluaciones de conflicto } \\
\text { cognitivo } \\
\text { c) Generar o introducir } \\
\text { nuevas ideas } \\
\text { d) Evaluar la } \\
\text { potencialidad de las } \\
\text { nuevas ideas }\end{array}$ \\
\hline $\begin{array}{l}3^{*} \text { Frase } \\
\text { Aplicación }\end{array}$ & $\begin{array}{l}\text { a) Demostrar en la } \\
\text { práctica los presupuestos } \\
\text { teoricos } \\
\text { b) Resolver problemas } \\
\text { tesricos yto prácticos } \\
\text { c) Ampliar los } \\
\text { conocimientos } \\
\text { introducidos }\end{array}$ & $\begin{array}{l}\text { a) Uitilizar las nuevas } \\
\text { ideas en diferentes } \\
\text { situaciones (conocidas y } \\
\text { novedosas) }\end{array}$ \\
\hline $\begin{array}{l}4^{\mathbf{r}} \text { Fase } \\
\text { Conclusión }\end{array}$ & $\begin{array}{l}\text { a) Resumir (resallar) las } \\
\text { principales ideas } \\
\text { b) Repasar el contenido } \\
\text { de la unidad }\end{array}$ & $\begin{array}{l}\text { a) Revisar el cambio en } \\
\text { las ideas } \\
\text { b) Mostrar el proceso de } \\
\text { E/A seguido }\end{array}$ \\
\hline
\end{tabular}

definido el esquema conceptual utilizando mapas de conceptos vuelve a ser útil. Creemos, como dice Novak, que «el mapa nos muestra posibles rutas de aprendizaje» y son con ellas con las que podemos hacer corresponder posibles secuencias de enseñanza. La elección de una u otra debe ser coherente con la necesidad de partir de las ideas previas de los alumnos y, por tanto, con los resultados del análisis didáctico.

Dependiendo de la amplitud y complejidad de la UD, puede ser necesario que su desarrollo requiera una o más secuencias de enseñanza o bien que la secuencia incluya fases reiterativas (AAVV 1987a, 1987b, Valcárcel et al. 1990), como por ejemplo: Iniciación 1- Información 1Iniciación 2- Información 2- Aplicación- Conclusión.

En el aula, el desarrollo de la UD ocurre a través de un conjunto de actividades de enseñanza. Entendemos por actividad cualquier tarea diferenciada que se realiza en clase por el profesor o los alumnos en relación con los objetivos didácticos. Así, por ejemplo, el planteamiento y resolución de problemas, la explicación del profesor, la puesta en común, un trabajo de consulta bibliográfica, la resolución de cuestiones en equipo... son actividades de enseñanza. Cada actividad tiene un contenido y una intencionalidad que la hacen diferente, y son estos indicadores los que deben guiar nuestra selección.

Respecto al contenido, son los objetivos didácticos qué nos marcamos los que nos indicarán qué actividades debemos seleccionar. La relación entre objetivos didácticos y actividades no es biunívoca, pues es posible que un objetivo requiera la selección de varias actividades o que una actividad persiga varios objetivos. La relación objetivos-actividad debe plantearse globalmente.

La intencionalidad de la actividad vendrá determinada por la fase de la secuencia de enseñanza a la que se destina, lo que puede hacer que un mismo contenido se plantee con diferentes niveles de información, o dirigismo, para el alumno. Esta segunda consideración hace que las posibilidades sean diversas; por ejemplo, la solubilidad de sólidos en líquidos es un hecho experimental sobre el que pueden plantearse actividades diferentes para las fases de iniciación, información y aplicación.

Un libro, un vídeo, un termómetro, una lámina... son materiales de aprendizaje que utilizamos en diferentes actividades. Aunque generalmente son estos conceptos los que asociamos con el de «material de aprendizaje», vamos a referimos exclusivamente a aquellos materiales escritos en Ios que se concreta la estrategia didáctica que estamos planificando y que llevaremos al aula.

Los materiales de aprendizaje que utilicemos deben mostrar claramente la estrategia didáctica del profesor, pues van a ser Ios instrumentos mediante los cuales el profesor comunica tanto el contenido de su enseñanza como su concepción. Aunque lógicamente no creemos que deba descartarse ninguna opción de antemano, oque sea posible generalizar sobre lo que resulta más o menos conveniente, proponemos que la estrategia didáctica del profesor se articule en torno a tres materiales de aprendizaje: el programa-guía, las hojas de trabajo y el cuaderno del alumno.

El «programa-guía» nos describe la secuencia de enseñanza en términos genéricos, relatando el conjunto de actividades incluidas en ellas (Gil et al. 1991). Debe concebirse como una propuesta de desarrollo de la UD o lección, y como tal debe ser abierto y sujeto a las modificaciones que se requieran durante su desarrollo como la supresión de una actividad prevista o la inclusión de una no prevista. El nivel de explicitación en la elaboración de este material dependerá de si se concibe como de uso exclusivo para el profesor o también para los alumnos.

Las «hojas de trabajo» amplían el contenido de la actividad y dan indicaciones para su desarrollo. Deben proporcionar información clara sobre los diferentes aspectos que se requieran para su ejecución, tales como el objeto de la actividad, los materiales que se precisan, el procedimiento a seguir, cuestiones a resolver... Lógicamente, no es posible generalizar sobre el contenido de las hojas de trabajo, pues dependerá tanto de la intencionalidad como del contenido de la actividad.

Respecto a la necesidad de las hojas de trabajo para Ilevar a cabo las actividades que aparecen en el progra. ma-guía, resulta evidente su necesidad, por ejemplo cuando se realizan en equipo, pues, al margen de orientaciones verbales, deben darse indicaciones por escrito para que puedan consultarse cuando surjan dudas o 
diferencias sobre su realización y para garantizar la autonomía de los equipos. En general, este material debe favorecer el seguimiento de la información que se intercambia en el aula (conclusiones de puestas en común, explicaciones del profesor...). Por tanto, las hojas de trabajo son un documento de uso del alumno que se requieren o son adecuadas para la ejecución de una actividad o para complementarla, asegurando la igualdad de información para todos los alumnos.

EI «cuaderno del alumno» es un material que se utiliza fundamentalmente con el objeto de personalizar el proceso de E/A y describir cómo se construye el conocimiento. No es una «libreta de trabajo» que la use el alumno para la realización sólo de actividades complementarias. Lo entenderros como el dosier que el alumno irá construyendo durante el desarrollo de la UD y, por tanto, debe ir incluyendo la información procedente de diferentes fuentes: profesor, equipo, clase, bibliografía, sus juicios de valor impresiones personales. Creemos que la importancia de este material es capital por ser el puente de unión entre el proceso colectivo mediante el que ocurre Ia enseñanza y el proceso individual exclusivo del aprendizaje.

V. La selección de estrategias de evaluación es la última tarea del modelo. Tanto las valoraciones como las consiguientes decisiones, implícitas en la evaluación, están condicionadas por la concepción que el profesor tenga del proceso de E/A.

Asumida como objetivo de la labor docente la construcción de aprendizajes por los alumnos, la evaluación, como un elemento más de esa labor, no puede sustraerse a dicho objetivo y hemos de considerarla, no con carácter restrictivo de valoración al final del proceso, sino con carácter formativo (Miras y Solé 1990), siendo por tanto su función la de favorecer dichos aprendizajes: ayudar para seguir avanzando y rectificar si es necesario. Desde la concepción constructivista, el profesor al valorar una situación, un hecho, un concepto o una experiencia, debe hacerlo de manera compartida con el alumno, mostrándole la utilidad que dicha valoración puede tener para el futuro o en sí misma, con objeto de que la evaluación sea realmente formativa. Lo contrario, es decir, hacerlo de manera unilateral y concluyente por parte del profesor es reforzar la idea de la evaluación como un proceso exclusivo de control.

Para que la evaluación sea formativa debemos utilizarla como un medio que nos proporcione información, tanto para suministrar una retroalimentación adecuada a los alumnos como para mejorar la enseñanza deI profesor. Se conviette, por tanto, en un instrumento para el seguimiento y al aprendizaje de los alumnos y para el seguimiento y mejora del desarrollo de la UD en el aula (MEC 1989).

Desde la planificación de la UD hemos de reflexionar y tomar decisiones sobre el qué, cómo y cuándo evaluar. Delimitar los aspectos relevantes que van a constituir el contenido de la evaluación es la primera decisión. Aunque son muchos los posibles elementos humanos y contextuales que podrían diferenciarse en torno al profe- sor, altumnos, recursos, centro, medio físico... en la planificación de una UD hay dos aspectos ineludibles íntimamente relacionados, el relativo a los aprendizajes de los alumnos y el relativo al desarrollo de la UD en eI aula.

En relación con los aprendizajes de los alumnos, la función formativa de la evaluación hace necesario como contenidos de la misma: a) la situación de partida, es decir, el conocimiento e ideas previas de los alumnos, b) los progresos en la construcción de conocimientos y del cambio conceptual que llevan a cabo los alumnos, y c) Ios conocimientos científicos adquiridos (conceptuales, procedimentales y actitudinales) (Coll et al. 1992).

La valoración de este contenido requiere que se hagan respecto a criterios de aprendizaje (Satterly y Swann 1988). Éstos deberán ser coherentes con la selección de objetivos realizada anteriormente.

En relación con el desarrollo de la UD en el aula son muchos los aspectos que podemos valorar, ya que es un proceso en el que interaccionan múltiples factores (ambiente de aula, interacción profesor-alumno y alumnoalumno, motivación, actitud ante la asignatura... ). Sin embargo, proponemos centrar nuestra atención en dos de ellos, las actividades seleccionadas y los materiales de aprendizaje utilizados (programa-guía, hojas de trabajo y cuaderno del alumno). Considerar prioritariamente éstos no es porque los otros carezcan de importancia, sino por ser actividades y materiales donde se concreta más directamente lo que el profesor piensa hacer en el aula, sirviéndonos su valoración para revisar, y en su caso modificar, aspectos concretos del diseño realizado.

Al igual que antes, la valoración requiere adoptar criterios que nos permitan obtener información sobre la adecuación de actividades y materiales a los objetivos que pretendemos alcanzar con los alumnos. La motivación e interés que genera en los alumnos, la complejidad de ejecución, Ia participación de los alumnos, la adecuación al tiempo previsto, el logro de los objetivos... pueden ser criterios adecuados para valorar las actividades de enseñanza. La comprensión de la información por el alumno, el nivel de autonomía en el trabajo en equipo, la funcionalidad y rentabilidad de los materiales, la capacidad dinamizadora del trabajo individual o en grupo... pueden ser criterios adecuados para valorar los materiales de aprendizaje.

La siguiente decisión en el procedimiento que proponemos se refiere a cómo y cuándo evaluar. Puesto que el contenido de la evaluación es diverso, también lo será el tipo de actividades que podamos llevar a cabo y los momentos a lo largo de la secuencia de enseñanza de la UD en los que podemos recabar esa información. Respecto a cuándo evaluar la intencionalidad de las diferentes fases incluidas en la secuencia de enseñanza que definamos (iniciación, información...) nos indicará el momento adecuado para el contenido de evaluación seleccionado. Así, por ejemplo, las ideas previas de los alumnos, las diagnosticaremos en la fase cuyo objetivo es la explicitación; el cambio conceptual exigirá el contraste entre esta fase y la de revisión... De iguai 
modo, la evaluación de actitudes científicas como críti$\mathrm{ca}$, respeto por las ideas ajenas, juicios de valor fundamentados... pueden hacerse en la puesta en común y debates.

Respecto a cómo evaluar, el carácter de la información deseada condicionará su recogida (actividad, instrumento). Sin embargo, es deseable y necesario, desde la función asignada a la evaluación, que las actividades de evaluación sean las propias actividades de enseñanza (trabajos prácticos, puestas en común, trabajos individuales...) o que se incorporen cuando la situación Io requiera, por ejemplo, en el caso de los exámenes. Pero incluso en esta situación es necesario que la evaluación tenga carácter formativo, por lo que es necesario que los alumnos tengan la posibilidad de discutir las respuestas, rehacer el examen, debatir las calificaciones... (Gil et al. 1991).

La última decisión dentro de la tarea es el diseño de instrumentos para la recogida de información. Especial importancia tiene el cuaderno del alumno, pues dado su carácter inđividual será el instrumento que mayor información nos dará sobre la implicación del alumno en el proceso de E/A. También es posible que diseñemos instrumentos específicos como pruebas escritas sobre contenidos puntuales, examen glogal de la UD..., o protocolos de observación con los que estimemos la progresión o adquisición de determinados conocimientos, como por ejemplo los derivados del trabajo colectivo o del empleo de instrumentos de medida (balanza, probeta...).

\section{CONSIDERACIONES FINALES}

Queremos terminar planteando Ia utilidad que puede tener para el profesor el disponer de unas estrategias de planificación sistematizadas como las que aporta este modelo. La toma de decisiones «preactivas» durante el proceso de planificación, el contraste de su adecuación durante la acción educativa y el volver a tomar decisio-

\section{REFERENCIAS BIBLIOGRÁFICAS}

AAVV, 1987a. Approaches to Teaching Energy. CLIS in the classroom (Centre for Studies in Science and Mathematics Education. University of Leeds).

AAVV, 1987b. Approaches to Teaching the Particulate Theory of Matter. CLIS in the classroom (Centre for Studies in Science and Mathematics Education. University of Leeds).

ALIBERAS, J. et al., 1989. La didáctica de las Ciencias: Una empresa racional, Enseñanza de las Ciencias, 7, pp. $277-284$ nes «postactivas» como consecuencia del proceso de evaluación seguido es un marco óptimo para plantearse y realizar investigaciones cuyos resultados tendrán una repercusión directa en la actividad docente del profesor, además de poder ser útil al resto de la comunidad de profesores. Creemos, por tanto, que en primera medida el profesor debe asumir su doble labor docente e investigadora (Elliot 1990) y que será la adquisición de conocimientos teóricos y metodológicos adecuados los que le permitan resolver los problemas que se plantee.

Creemos que el papel del profesor como "constructor" de su proceso de enseñanza está garantizado si en vez de reproducir un plan de instrucción, ajeno a sus teorías, creencias, contexto..., en el que encontrará aspectos en desacuerdo, lleva a cabo una planificación bajo los presupuestos del modelo, evidentemente considerando toda la información (textos científicos, trabajos sobre ideas de los alumnos, materiales de aprendizaje y propuestas didácticas ya elaboradas, instrumentos de evaluación...) que facilite la realización de las cinco tareas anteriores. De igual modo, creemos necesario que el profesor adopte este papel de generador de su enseñanza si en su interés está la implantación real de la reforma educativa, pues un análisis del DCB evidencia que Ia concepción constructivista sobre el proceso de E/A es el eje fundamental de sus planteamientos y difícilmente podremos, los profesores, llevar al aula dichos planteamientos para que el alumno construya su conocimiento científico si previamente, cada profesor no actúa constructivamente desde la planificación de su enseñanza, implicándose en un proceso de explicitación de sus iđeas y contrastación en el aula, de manera que pueda ir modificando sus planteamientos teóricos y adecuándolos a su contexto real.

\section{NOTA}

Este trabajo forma parte del proyecto de investigación financiado por la DGICYT, número de referencia PB 87-0683.

CHALMERS, A., 1982. ¿Qué es esa cosa llamada Ciencia? (Siglo XXI: Madrid).

CLARK, C.M. y PETERSON, P.L., 19.90. «Procesos de pensamiento de los docentes, en Wittrock, M.C. (ed.), La investigación de la enseñanza, $I I$. Profesores y alumnos. (Paidós: Barcelona).

CLARK, C.M. y YINGER, R, 1979. "Three studies of teacher planning». East Lansing: Institute for research on teaching. Michigan State University. Research Series, 55. 
COLL, C., 1986. Bases Psicológicas, Cuadernos de Pedagogía, 139, pp. 12-16.

COLL, C., 1987. Psicología y curriculum. (Laia: Barcelona).

COLL, C., 1988. Significado y sentido en el aprendizaje escolar. Reflexiones en torno al concepto de aprendizaje significativo, Infancia y Aprendizaje, 41, pp. 131-142.

COLL, C. y SOLÉ, I., 1987. La importancia de los contenidos en la enseñanza, Investigación en la Escuela, 3, pp. 19-26.

COLL, C. et al., 1992. Los contenidos de la reforma. Enseñanza y aprendizaje de conceptos, procedimientos y actitudes. (Santillana: Madrid).

COSGROVE, M. y OSBORNE, R., 1991. Modelos didácticos para cambiar las ideas de los alumnos, en Osborne R. y Freyberg P. (eds), El aprendizaje de las Ciencias. Implicaciones de la Ciencia de los alumnos. (Narcea: Madrid).

DRIVER, R., 1986. Psicología cognoscitiva y esquemas conceptuales de los alunınos, Enseñanza de las Ciencias, 4, pp. 3-15.

DRIVER, $R$, 1988. Un enfoque constructivista para el desarrollo del cunículo en Ciencias, Enseñanza de las Ciencias, 6, pp. 109-121.

DRIVER et aI., 1989. Ideas cientificas en la infancia y la adolescencia. (Morata-MEC: Madrid).

ELLIOT, J., 1990. Lo investigación-acción en educación (Morata: Madrid).

GARCIA-ESTAÑ, R, et al., 1988. El aprendizaje de la Fisica en EGB. Exploración diagnóstica en Murcia (ICE: Murcia).

GIL, D., 1986. La metodología científica y la enseñanza de las Ciencias. Unas relaciones controvertidas, Enseñanza de las Ciencias, 4, pp. $111-121$.

GIL, D. y MARTÍNEZ, J., 1987. Los programas-guía de actividades: una concreción del modelo constructivista del aprendizaje de las Ciencias, Investigación en la Escuela, 3, pp. 3-12.

GIL, D, et al., 1991. La enseñanza de las cienciasen la educación secundaria. (ICE-Horsori: Barcelona).

GIORDAN, A. y VECCHI, G., 1988. Los origenes del saber. De las concepciones personales a los conceptos científicos. (Diada Editores: Sevilla).

HERNÁNDEZ, F, 1989. El lugar de los procedimientos, Cuadernos de Pedagogín, 172, pp. 60-64.

HODSON, D., 1988a. Towards a phylosophicaliy more valid Science curriculum, Science Education, 72, pp. 19-40.

HODSON, D., 1988b. Filosof́a de la ciencia y educación científica, en Porlan, R. et al. (comp.), Constructivismo y enseñanza de las ciencias. (Diada: Sevilla).

HEWSON, P.W. y HEWSON, M.G., 1988. An appropriate conception of teaching science: a view from studies of science learning, Science Education, 72, pp. 597-614.
HIERREZUELO, J. y MONTERO, A., 1988. La ciencia de los alumnos. (Laia-MEC: Barcelona).

LINN, M.C., 1987. Establishing a research base for Science Education: challenges, trends an recommendations, Journal of Research in Science Teaching, 24, pp, 191-216.

MEC., 1989. Diseño Curricular Base. Educación Secundaria Obligatoria (MEC: Madrid).

MIRAS, M. y SOLÉ, I., 1990. La evaluación del aprendizaje, en Coll, C., Palacios, J, y Marchesi, A. (eds.), Desarrollo psicológico y educación II. Psicología de la Educación. (Alianza: Madrid).

NOVAK, J.D. y GOWIN, D.B., 1988. Aprendiendo a aprender. (Martínez Roca: Barcelona).

NUSSBAUM, J. y NOVICK, S., 1982. Alternative frameworks, conceptual conflict and accommodation: toward a principled teaching strategy, Instructional Science, 11, pp. 183-200.

OSBORNE, R. y WITTROCK, M., 1985. The generative leaning model and its implications for Science Education, Studies in Sciencie Education, 12, pp. 59-87.

POSNER, G., et al. 1982. Accommodation of a scientific conception: toward a theory of conceptual change, Science Education, 66, pp. 211-227.

POZO, J.I., 1990. Teorías cognitivas del aprendizaje. (Morata: Madrid).

POZO, J.I., et al., 1991. Las ideas de los alumnos sobre la Ciencia: una interpretación desde la psicología cognitiva, Enseñanza de las Ciencias, 9, pp. 83-94.

POZO, J.I., 1992. El aprendizaje y la enseñanza de hechos y conceptos, en Coll, C., Palacios, J. y Marchesi, A. (eds.) Desarrollo psicológico y educación II. Psicología de la Educación. (Alianza: Madrid).

SALINAS, D., 1990. Los profesores y la planificación de la enseñanza: ¿qué hagoel lunes?, Cuadernos de Pedagogía, 184, pp. 96-101.

SANMARTÍ, N. et al, 1990. Los procedimientos (Área Ciencias de la Naturaleza), Cuadernos de Pedagogia, 180, pp. 28-32.

SARABIA, B., 1992. El aprendizaje y la enseñanza de las actitudes, en Coll, C., Palacios, J. y Marchesi, A. (eds.) Desarrollo psicológico y educación $I T$. Psicología de la Educación. (Alianza: Madrid).

SATTERLY, D. y SWANN, N., 1988. Los exámenes referidos al criterio y al concepto de Ciencias: un nuevo sistema de evaluación, Enseñanza de las Ciencias, 6, pp. 278-285.

SCOTT, P., 1987. A constructivist view for learning and teaching in Science. CLIS in the classroom (Centre for Studies in Science and Mathematics Education. University of Leeds).

SHAYER, M. y ADEY,P., 1984. La Ciencia de enseñar Ciencia. (Narcea: Madrid).

VALCÁRCEL, M. V. et al., 1990. Problemática didáctica del aprendizaje de las Ciencias Experimentales. (Servicio de Publicaciones Universidad de Murcia). 